\title{
Stimulus articulation and backward learning
}

DAVID T. HAKES

UNIVERSITY OF TEXAS

\begin{abstract}
Abstraet
An eight-pair PA list of low-meaningfulness paralogs was learned to a criterion of two successive errorless trials by two groups of Ss, one of which articulated the stimuli while the other did not. All Ss then learned the same pairs in the backward direction for 16 trials, half of each group articulating the stimuli. Stimulus articulation had no effect on first-list learning. First-list articulation facilitated backward recall, the effect diminishing as backward learning progressed.

\section{Problem}

Schulz \& Tucker (1962a) have shown that requiring Ss to articulate unfamiliar stimuli during PA learning can retard learning. Since $S$ has a limited amount of time (e.g., 2 sec.) during which to anticipate the response, articulating stimuli should decrease the effective anticipation interval. If the latency with which a response is emitted is a function of the S-R associative strength, it may be predicted that decreasing the anticipation interval will retard learning as the association must be stronger if $\mathrm{S}$ is to emit the response within the reduced interval. That the effect of articulation is attributable to a reduction of the effective anticipation interval is indicated by the results of a second experiment (Schulz \& Tucker, 1962b) in which lengthening the anticipation interval to $4 \mathrm{sec}$. eliminated the stimulus articulation effect.

It should be noted, however, that an earlier experiment by Hunt (1959) found stimulus articulation to have no effect on PA learning. Since the procedures used and analyses performed were somewhat atypical, Hunt's results should probably be considered as only suggestive.

The present experiment was performed in an attempt to replicate Schulz \& Tucker's finding concerning stimulus articulation. To provide further information on the effects of stimulus articulation, a backward learning task was included. It may be expected that requiring stimulus articulation would force Ss to learn more about the stimuli than if articulation were not required. Thus, the stimuli should be more available for recall when articulation is required.

\section{Method}

Two groups learned a PA list of low-meaningfulness paralogs, one group being required to articulate each stimulus before anticipating the response. Following learning to a fixed criterion, all Ss learned the same PA list in the backward direction. For the B-A learning task, half of each group articulated the stimuli. Thus, with respect to B-A learning, the design constitutes a
$2 \times 2$ factorial, with first- and second-list stimulus articulation the variables. A total of $40 \mathrm{Ss}(\mathrm{N}=10 \mathrm{per}$ cell) served in the experiment and were assigned to conditions according to a random sequence. Four Ss were discarded, all for failure to learn the first list within 52 trials; each was replaced by the next $S$ to appear.

Sixteen dissyllabic paralogs (CVCVC) were selected from the low meaningfulness end of Taylor's (1959) scale. Meaningful and formal similarity among the items were as low as careful inspection would permit. The items were randomly combined to form eight pairs.

For the first-list, non-articulation condition, Ss were instructed to anticipate each response as soon as the stimulus appeared. For the articulation condition, they were instructed to pronounce the stimulus before attempting to anticipate. Both groups were instructed to pronounce the response when it appeared if they had failed to anticipate it and to correct themselves if they had anticipated incorrectly. Since many of the items yielded a variety of pronunciations, this correction procedure avoided the problem of arbitrarily deciding upon a "correct" pronunciation. If, after anticipating, $S$ did not correct himself, the response was accepted as correct.

Following instructions, the PA list was presented in the $\mathrm{A}-\mathrm{B}$ direction and learning carried to a criterion of two successive errorless trials. Following a 2-min. interval, during which the list was changed and additional instructions read, the list was presented in the B-A direction for 16 trials. The B-A learning instructions required half of each group to articulate stimuli and required all Ss to anticipate on the first trial. The Ss were informed that the second list contained the same pairs as the first and that each pair was presented backwards.

Both lists were presented on a memory drum at a $2: 2-\mathrm{sec}$. rate with a $4 \mathrm{sec}$. intertrial interval. Five random orders of each list were used.

\section{Results}

Numbers of trials to reach a criterion of two successive errorless trials were analyzed for the A-B list. The means for the articulation and non-articulation conditions were 31.35 and 32.10 trials respectively. In the analysis, neither first- nor second-list articulation nor the interaction approached significance, all Fs $<1$, indicating that articulation did not affect $A-B$ learning and that the groups given different second list treatments could be considered equivalent. Inspection of the 
Table 1

Mean Number of Correct Responses in B-A Recall as a Function of Stimulus-Articulation Conditions

\begin{tabular}{clccc} 
& \multicolumn{3}{c}{ First List } \\
& & \multicolumn{2}{c}{ Articulation } & Non-Articulation \\
& & & & \\
Articulation & M & 2.40 & 1.30 \\
Second List & SD & 1.51 & 0.95 \\
Non-Articulation & & & & \\
& SD & 3.20 & 2.10 \\
& & 2.04 & 1.73
\end{tabular}

learning curves suggests that articulation did not greatly affect A-B learning at any point.

Since Ss were required to anticipate on the first trial of B-A learning, this trial may be considered a backward-recall trial. The mean numbers of correct responses recalled by the four groups are presented in Table 1. Analysis of the data indicated that firstlist articulation produced a significant effect, $F(1,36)=4.69 ; p<.05$. Second-list articulation did not reach significance, $F(1,36)=2.48, p>.05$, nor did the interaction, $F<1$. The first-list articulation effect indicates that $S s$ required to articulate during $A-B$ learning recalled more correct $\mathrm{B}-\mathrm{A}$ associations than those who did not.

Numbers of correct responses per two-trial block were analyzed over the 16 trials of B-A learning. The only main effect producing a signficant difference was trial blocks, $F(7,252)=82.25 ; \mathrm{p}<.001$. The only interaction to reach significance was the triple interaction, Trial Blocks $x$ First-List Articulation $x$ Second-List Articulation, $F(7,252=2.09, p<.05$. This indicates that the effect of first-list articulation diminished as learning progressed.

To further explore the effects of first-list articulation, an analysis of the response-learning stage during $\mathrm{B}-\mathrm{A}$ learning was performed. First-list articulation approached significance, $F(1,36)=2.70 ; p<.10$. Neither second-list articulation nor the interaction approached significance, both Fs $<1$.

Finally, an analysis was performed of responses (A-terms) which were misprounced during the first five trials of B-A learning. The number of mispronounciations was taken as a proportion of the total overt errors made during these trials. For Ss who articulated during first-list learning the proportion was .21 , and for Ss who did not articulate it was $.34, F(1,38)=439.32$, $\mathrm{p}<.001$. Thus it would appear that first-list articulation not only resulted in greater availability of the A-terms for backward recall but also resulted in these terms becoming better integrated.

\section{Diseussion}

The results of the present experiment provide little support for Schulz \& Tucker's conclusion that stimulus articulation affects rate of PA learning. There is no immediately apparent reason for the lack of agreement between the results of the two experiments. The procedures used appear to have been highly similar, as were the materials. While a five-pair list was used in the earlier experiment and an eight-pair list here, it is difficult to attribute the difference in results to this difference. It might be expected, rather, that increasing list-length might increase the effect of articulation.

It is apparent that articulation does not produce large differences in stimulus availability. The articulation effect diminished rapidly during B-A learning and also yielded no difference in the response-learning stage. This latter finding is, however, interpretable in another manner. The variable measured in an analysis of the response-learning stage is the trial on which each response first occurs, either correctly or as an intrusion. This indicates only that the response was integrated and available by that trial and not that is was not available before that trial. Thus the response-learning analysis may simply be an insensitive measure of the point at which an item becomes available. This interpretation is supported by the analysis of mispronounced responses, which would appear to provide a more sensitive measure of non-availability of an integrated response.

Finally, it should be noted that while the stimuli were apparently made more available as a result of having been articulated $\mathrm{B}-\mathrm{A}$ recall was considerably less than perfect $(35 \%)$ at a point at which the A-B associations had been learned to a high criterion.

\section{References}

Hunt, R. G. Meaningfulness and articulation of stimulus and response in paired-associate learning and stimulus recall. $J$. exp. Psychol., 1959, 57, 262-267.

Schulz, R. W., \& Tucker, I. F. Stimulus familiarization in pairedassociate learning. J. exp. Psychol., 1962a, 64, 549-550.

Schulz, R. W., \& Tucker, I. F. Stimulus familiarization and length of the anticipation interval in paired-associate learning. Psychol. Rec., 1962b, 12, 341-344.

Taylor, J. D. The meaningfulness of 320 words and paralogs. Unpublished doctoral dissertation, Duke University, 1959. 